\title{
Expression of CRISPR/Cas single guide RNAs using small tRNA promoters
}

ADAM L. MEFFERD, ANAND V.R. KORNEPATI, HAL P. BOGERD, EDWARD M. KENNEDY, and BRYAN R. CULLEN

Department of Molecular Genetics \& Microbiology and Center for Virology, Duke University Medical Center, Durham, North Carolina 27710, USA

\begin{abstract}
The in vivo application of CRISPR/Cas-based DNA editing technology will require the development of efficient delivery methods that likely will be dependent on adeno-associated virus (AAV)-based viral vectors. However, AAV vectors have only a modest, 4.7-kb packaging capacity, which will necessitate the identification and characterization of highly active Cas9 proteins that are substantially smaller than the prototypic Streptococcus pyogenes Cas9 protein, which covers $\sim 4.2 \mathrm{~kb}$ of coding sequence, as well as the development of single guide RNA (sgRNA) expression cassettes substantially smaller than the current $\sim 360$ bp size. Here, we report that small, 70-bp tRNA promoters can be used to express high levels of tRNA:sgRNA fusion transcripts that are efficiently and precisely cleaved by endogenous tRNase $Z$ to release fully functional sgRNAs. Importantly, cells stably expressing functional tRNA:sgRNA precursors did not show a detectable change in the level of endogenous tRNA expression. This novel sgRNA expression strategy should greatly facilitate the construction of effective AAV-based Cas9/sgRNA vectors for future in vivo use.
\end{abstract}

Keywords: CRISPR/Cas; tRNase Z; gene editing; tRNA processing

\section{INTRODUCTION}

The prospect that in vivo genome editing might emerge as a feasible treatment for a range of human diseases has been the focus of considerable recent interest, yet many problems remain to be resolved before genome editing can become clinically useful (Cox et al. 2015). The most promising genome editing technology is based on the programmable Cas9 endonucleases derived from bacterial CRISPR loci (Hsu et al. 2014). Cas9 proteins can be guided to genomic DNA targets by a single guide RNA (sgRNA) consisting of a variable domain complementary to the DNA target and a structured scaffold domain derived from the bacterial crRNA (CRISPR RNA) and tracrRNA (trans-activating crRNA) molecules. A key advantage of the CRISPR/Cas technology is that the Cas9 protein remains invariant yet can be readily reprogrammed to cleave novel DNA target sites by expressing an sgRNA with a different variable domain.

Current CRISPR/Cas vectors generally rely on the Cas9 protein encoded by Streptococcus pyogenes (Spy), which is encoded by an 4.2-kb gene (Cong et al. 2013; Mali et al. 2013b; Hsu et al. 2014). However, as discussed previously by ourselves and others (Cox et al. 2015; Kennedy et al. 2014), effective delivery of both the Cas 9 gene and cognate sgRNAs into

Corresponding author: bryan.cullen@duke.edu

Article published online ahead of print. Article and publication date are at http://www.rnajournal.org/cgi/doi/10.1261/rna.051631.115. tissues in vivo will likely require the development of adenoassociated virus (AAV)-based vectors, as only AAV can be readily produced at titers sufficient to transduce enough cells in vivo to exert the desired phenotypic effect (Kotterman and Schaffer 2014). However, AAV vectors have a DNA packaging limit of $\sim 4.7 \mathrm{~kb}$ of which $\sim 290$ bp must be dedicated to the two invariant AAV inverted terminal repeats (ITRs), leaving only $\sim 4.4 \mathrm{~kb}$ as the payload capacity. To circumvent this problem, Swiech et al. (2015) generated an AAV vector expressing the $\sim 4$. 2 -kb Spy Cas 9 gene using a minimal promoter element and then used a second AAV to express a cognate sgRNA. This approach requires coinfection by each of these two AAVs in order to induce genome editing, which would clearly be less efficient in an in vivo setting. For this reason, there has been considerable interest in identifying highly active smaller Cas 9 proteins, encoded by other bacterial species. For example, both the Neisseria meningitidis (Nme) and Staphylococcus aureus (Sau) Cas9 genes are only $\sim 3.2 \mathrm{~kb}$ in size (Esvelt et al. 2013; Hou et al. 2013; Ran et al. 2015). However, this would still leave only $\sim 1.2 \mathrm{~kb}$ of space for the RNA polymerase II (Pol II) promoter and poly(A) addition site required for Cas9 expression as well as a nuclear

(C) 2015 Mefferd et al. This article is distributed exclusively by the RNA Society for the first 12 months after the full-issue publication date (see http://rnajournal.cshlp.org/site/misc/terms.xhtml). After 12 months, it is available under a Creative Commons License (Attribution-NonCommercial 4.0 International), as described at http://creativecommons.org/licenses/by$\mathrm{nc} / 4.0 /$. 
localization signal (NLS) required for Cas9 nuclear import, the sgRNA, and the RNA polymerase III (Pol III) promoter required for sgRNA transcription. In many cases, two or more sgRNAs would be desirable, for example, to allow versions of Cas9 mutated to cleave only one strand of a target DNA sequence, so-called Cas9 nickases, to induce DNA cleavage by nicking two closely adjacent sites on opposite strands of the DNA duplex (Cong et al. 2013; Mali et al. 2013a; Ran et al. 2013).

Previous work has focused on using the U6 Pol III promoter to drive sgRNA transcription (Cong et al. 2013; Mali et al. 2013b; Kennedy et al. 2014; Swiech et al. 2015). The U6 promoter, while very effective, is 254 bp long and two U6 promoters would therefore require more than $10 \%$ of the entire packaging capacity of an AAV vector. Moreover, the U6 promoter requires transcribed sgRNA sequences to initiate with a "G" residue, which either restricts the number of available targets or introduces a single base mismatch to the DNA target. It is therefore desirable to identify equally effective Pol III-dependent promoters that are both smaller than U6 and lack this sequence requirement. Here, we report that tRNA promoters, of human or viral origin, can be used to express high levels of sgRNAs specific for a wide range of DNA targets and bacterial Cas9 proteins.

\section{RESULTS}

We have previously demonstrated that mouse $\gamma$-herpesvirus 68 (MHV68) encodes several 60-nt long premicroRNA (pre-miRNA) molecules that are initially transcribed as a fusion transcript consisting of a $5^{\prime}$ viral tRNA moiety fused to a $3^{\prime}$ pre-miRNA hairpin (Bogerd et al. 2010). These precursor RNAs are then precisely separated into a $5^{\prime}$ tRNA and a $3^{\prime}$ pre-miRNA upon cleavage by cellular tRNase $Z$, which functions to define the $3^{\prime}$ end of cellular tRNAs. We have also demonstrated that human tRNAs, when fused to a pre-miRNA hairpin, give rise to both the pre-miRNA intermediate and a functional mature miRNA and that this again requires processing by endogenous tRNase $\mathrm{Z}$. We therefore wondered whether human tRNAs could also be used to generate sgRNAs via a precursor tRNA fusion intermediate, as schematically shown in Figure 1A. When compared with previously described tRNA:pre-miRNA fusion transcripts, this tRNA:sgRNA fusion differs in that the sgRNA is both significantly larger ( $\sim 101$ vs. $\sim 60 \mathrm{nt}$ ) and folded into a more complex secondary structure.

Initially, we generated fusion transcripts consisting of a human glutamine or histidine tRNA, or an MHV68 tRNA (M17), linked to previously described Spy Cas9 sgRNAs specific for the human papillomavirus serotype 18 (HPV-18) E6 or E7 gene (Kennedy et al. 2014). Indicator plasmids consisting of these viral DNA target sequences inserted $3^{\prime}$ to a translation initiation codon and $5^{\prime}$ to the firefly luciferase (FLuc) gene, constructed as previously described (Kennedy et al. 2014, 2015), were then cotransfected into $293 \mathrm{~T}$ cells along with a
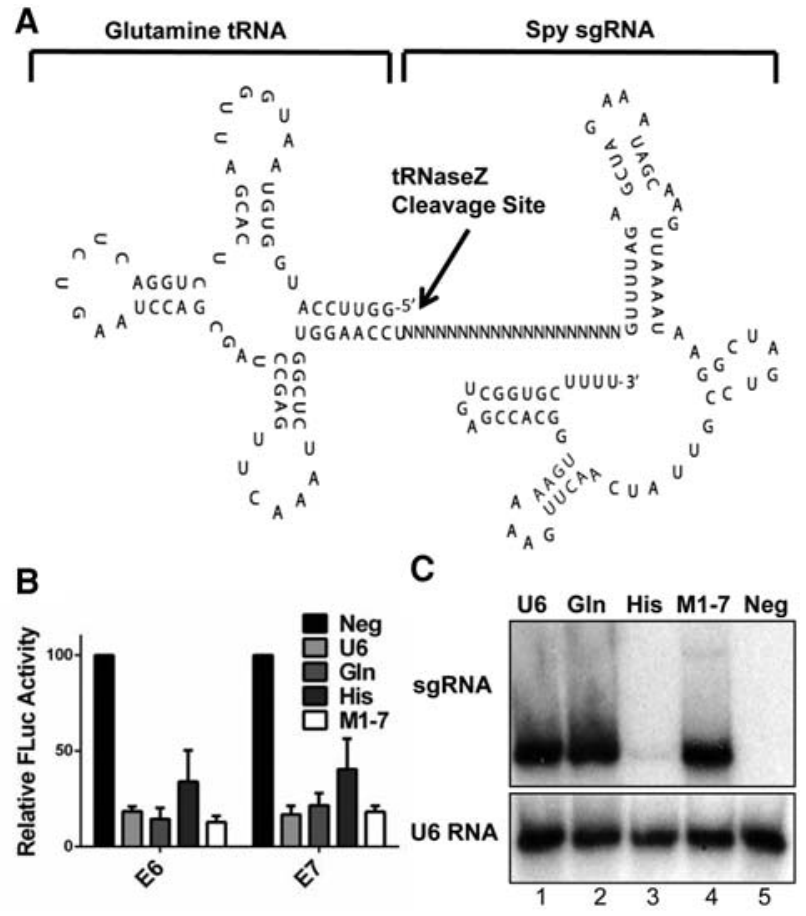

FIGURE 1. Streptococcus pyogenes sgRNAs can be expressed at similar levels using tRNA or U6 promoters. (A) Schematic of a human glutamine tRNA-sgRNA fusion with the predicted tRNaseZ cleavage site indicated. The "N" residues represent the variable sgRNA targeting sequence. (B) Relative luciferase activity in 293T cells cotransfected with plasmids expressing the Spy Cas9 protein and sgRNAs specific for the HPV-18 E6 or E7 genes or a negative control sgRNA (Neg), and their cognate indicator plasmids. sgRNAs were expressed from either the U6 promoter or the indicated tRNA. Average of three experiments with SD indicated. (C) Northern blot of Spy sgRNAs recovered from 293T cells transfected with a plasmid expressing Spy Cas9 and sgRNAs specific for the HPV-18 E7 gene expressed from either a U6 or tRNA promoter. Endogenous cellular U6 RNA served as a loading control.

plasmid encoding Spy Cas9 and an sgRNA linked to either a tRNA or U6 promoter as well as a Renilla luciferase (RLuc)based internal control plasmid. Cells were harvested at $72 \mathrm{~h}$ post-transfection and FLuc and RLuc levels determined. As shown in Figure 1B, we observed effective and comparable knockdown of both the E6- and E7-based indicator constructs by not only the U6 promoter-based sgRNA expression vector but also by the vectors based on the tRNA $\mathrm{GLN}_{\mathrm{GN}}$ and $\mathrm{tRNA}_{\mathrm{M} 1-7}$ promoters, while the $\mathrm{RNA}_{\mathrm{HIS}}$ vector appeared slightly less effective. Analysis of the predicted Cas9 cleavage site in the indicator plasmid, using the Surveyor endonuclease to detect introduced insertion or deletion (indel) mutations, detected such mutations in DNA recovered from the cultures cotransfected with all four sgRNA expression plasmids but not in the control culture, as expected (Supplemental Fig. S1).

We next analyzed the expression level of the E7-specific sgRNA in the transfected 293T cells using a Spy sgRNA scaffold-specific probe. As shown in Figure 1C, we saw comparable levels of the mature E7-specific sgRNA produced by the 
U6, $\mathrm{tRNA}_{\mathrm{GLN}}$ and $\mathrm{tRNA}_{\mathrm{M} 1-7}$-based vectors but far less from the $t R N A_{H I S}$-based vector. The fact that the $\mathrm{RNA}_{\mathrm{HIS}}$-based vector nevertheless gave rise to a significant repression of a cognate indicator plasmid (Fig. 1B) demonstrates that even low levels of a functional Cas9/sgRNA complex are able to exert a significant phenotypic effect in this cell-based assay. While we did not observe detectable levels of the predicted $\sim 175$-nt tRNA:sgRNA fusion transcript in the cultures transfected with the tRNA ${ }_{\mathrm{GLN}}$ or $\mathrm{tRNA}_{\mathrm{HIS}}$-based vectors, a trace amount was observed in the culture transfected with the tRNA $_{\mathrm{M} 1-7}$ based vector (Fig. 1C, lane 4).

To extend these studies to a distinct smaller Cas9 protein with a different sgRNA scaffold, we next analyzed the expression and function of Nme Cas9 sgRNAs transcribed using the U6 or tRNA promoters (Esvelt et al. 2013; Hou et al. 2013). The Nme sgRNA scaffold is entirely different in sequence from the Spy sgRNA scaffold and also somewhat larger in size. We again analyzed Cas9 activity against two different DNA targets, each of which consists of a bacterially derived, natural protospacer sequence called protospacer 9 (P9) or P25 (Zhang et al. 2013). The Nme Cas9 protospacer adjacent motif (PAM) used here was $5^{\prime}$-NNNNGATT- $3^{\prime}$, as previously reported (Hou et al. 2013).

As shown in Figure 2A, we again observed the specific knockdown of the cognate FLuc-based indicator plasmids in cotransfected cells, though this was less extensive than observed with Spy Cas9 (Fig. 1B). Northern analysis of sgRNA expression, using a probe specific for the Nme sgRNA scaffold, showed closely comparable levels of the P25-specific sgRNA in cells transfected with the U6, tRNA $\mathrm{GLN}_{\text {, and }}$ $\mathrm{tRNA}_{\mathrm{M1-7}}$-based vectors and again far lower levels with the

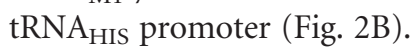

To extend these studies, and to test whether different sgRNA variable regions might interfere with tRNase Z-mediated processing of the tRNA:sgRNA fusion transcript, we next analyzed the expression of a wide range of Nme sgRNAs bearing either 25-nt (P25, P9, G6P) or 24-nt (ICP4, GFP) variable regions using either the $\mathrm{U} 6$ or $\mathrm{tRNA}_{\mathrm{GLN}}$ promoter. As may be observed (Fig. 2C), we saw generally closely comparable levels of sgRNA expression in all cases. In the case of the ICP4specific tRNA $\mathrm{GLN}_{\mathrm{G}}$ :sgRNA vector, we again noticed a trace amount of a larger transcript migrating at the predicted $\sim 200$-nt size of the initial tRNA:sgRNA fusion transcript (Fig. 2C, lane 6).

The Sau Cas9 gene shares the small $\sim 3.2 \mathrm{~kb}$ size of the Nme Cas9 gene and Sau Cas9 is highly active when presented with DNA targets that contain its cognate PAM sequence $5^{\prime}$ NNGRRT-3', where " $R$ " represents a purine residue (Ran et al. 2015). Therefore, Sau encodes a promising Cas 9 gene for potential use in AAV vectors. To analyze whether we could also produce active Sau sgRNAs using tRNA promoters, we expressed the identical Sau sgRNA, specific for a target within the HSV-1 ICP0 gene, using a wide range of human tRNAs. By indicator assay, we observed strong knockdown when using human $\mathrm{tRNA}_{\mathrm{GLN} 1}, \mathrm{tRNA}_{\mathrm{GLN} 2}, \mathrm{tRNA}_{\mathrm{GLY}}$,
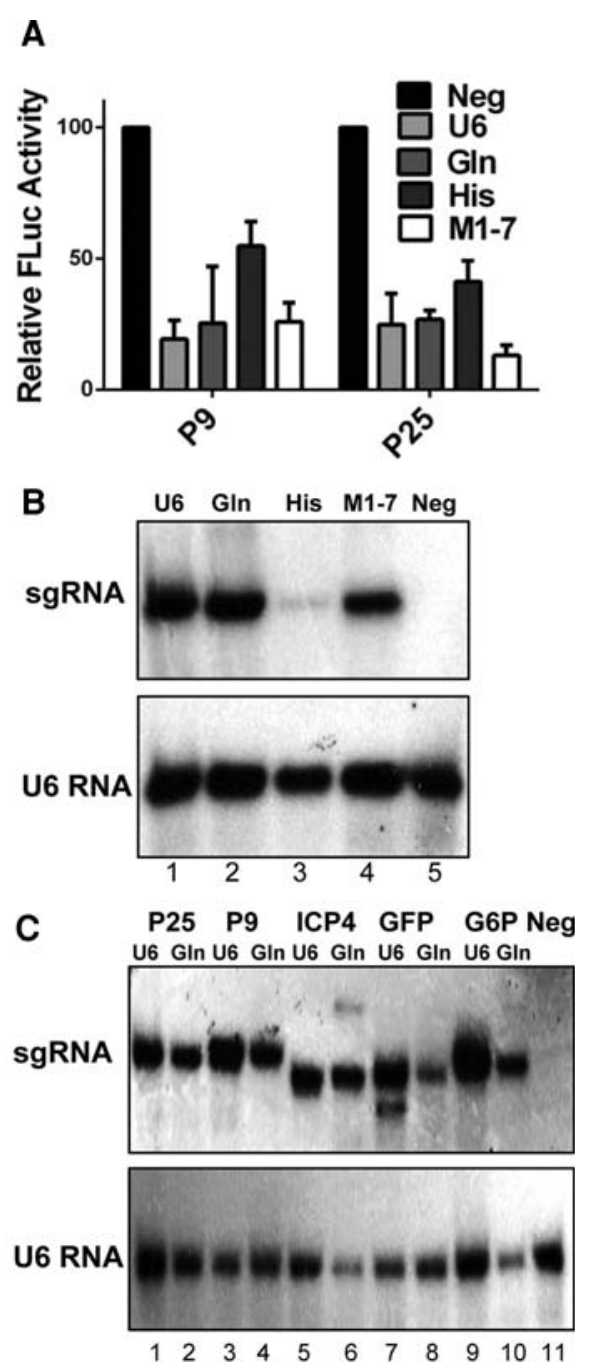

FIGURE 2. Neisseria meningitidis sgRNA expression using tRNA-derived promoters. $(A)$ Relative luciferase activity detected in $293 \mathrm{~T}$ cells cotransfected with plasmids expressing Nme Cas 9 and sgRNAs specific for protospacer 9 (P9) or 25 (P25) (Zhang et al. 2013) or a negative control sgRNA (Neg), and their cognate indicator plasmids. sgRNAs were expressed from either the U6 promoter or the indicated tRNA. Average of three experiments with SD indicated. (B) Northern blot of Nme sgRNAs recovered from 293T cells transfected with a plasmid expressing Nme Cas9 and sgRNAs specific for the P25 sequence expressed using U6 or the indicated tRNA. Endogenous cellular U6 RNA served as a loading control. $(C)$ Same as $B$ except that expression of a variety of distinct sgRNA guide sequences, specific for the indicated gene sequence, was analyzed. (P25) protospacer 25; (P9) protospacer 9; (ICP4) HSV-1 infected cell protein 4; (GFP) green fluorescent protein; (G6P) glucose-6-phosphatase.

$\mathrm{tRNA}_{\mathrm{GLU}}$, or $\mathrm{tRNA} \mathrm{PRO}_{\mathrm{PRO}}$ to drive sgRNA expression and readily detectable knockdown when using tRNA $_{\mathrm{ASN}}$ or tRNA $\mathrm{CYS}_{\mathrm{C}}$ but little or no detectable knockdown when using tRNA ${ }_{\text {TYR }}$ (Fig. $3 \mathrm{~A})$. Northern analysis, using a probe specific for the Sau sgRNA scaffold, showed high-level expression of the mature sgRNA with the $\mathrm{tRNA}_{\mathrm{GLN} 1}, \mathrm{tRNA}_{\mathrm{GLN} 2}, \mathrm{tRNA}_{\mathrm{GLY}}, \mathrm{tRNA}_{\mathrm{GLU}}$, and especially $t R N A_{P R O}$ promoters. Expression of the mature sgRNA was more modest with the $\mathrm{tRNA}_{\mathrm{GLY}}$, $\mathrm{RNA}_{\mathrm{CY}}$, and 

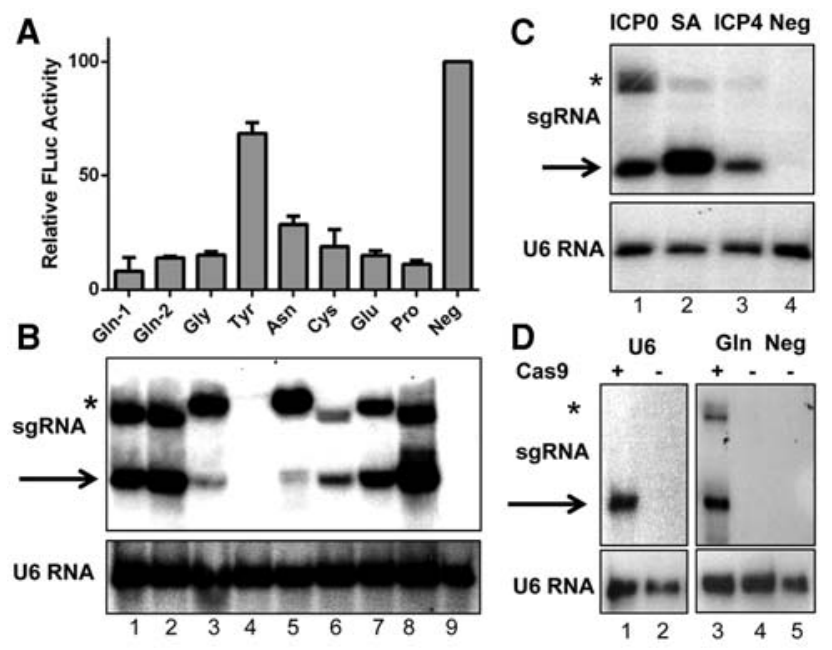

FIGURE 3. Various human tRNAs can be used to express $S$. aureus Cas9-specific sgRNAs. (A) Relative luciferase activity from 293T cells cotransfected with plasmids expressing the Sau Cas9 protein and sgRNAs specific for the HSV-1 ICP0 gene or a no sgRNA control (Neg), and a cognate ICP0-based indicator plasmid. sgRNAs were expressed using the various human tRNAs indicated. Average of three experiments with SD indicated. (B) Northern blot of Sau sgRNAs recovered from 293T cells transfected with a plasmid expressing Sau Cas9 and an sgRNA specific for HSV-1 ICP0. The sgRNAs were expressed using the indicated human tRNAs. Endogenous cellular U6 RNA served as a loading control. The arrow indicates the predicted location of the mature sgRNA, while the asterisk indicates the unprocessed tRNA:sgRNA fusion transcript. The latter varies in size depending on the size of the tRNA used. (C) Similar to $B$, except that this Northern analyzes the expression of sgRNAs transcribed using human tRNA $_{\mathrm{GLN} 1}$ that are specific for the HSV-1 ICP0 or ICP4 genes or the HBV surface antigen (SA) gene. (D) Northern blot analysis of Sau sgRNAs recovered from $293 \mathrm{~T}$ cells cotransfected with a plasmid expressing an sgRNA targeting HSV-1 ICP0 from either a U6 or tRNA $\mathrm{GLN}_{\mathrm{GL}}$ promoter and a plasmid expressing Sau Cas9 or an empty vector control. Endogenous cellular U6 RNA served as a loading control.

tRNA $_{\mathrm{ASN}}$ promoters, while the $\mathrm{tRNA}_{\mathrm{TYR}}$ promoter did not give rise to a detectable signal. Interestingly, and in contrast to what was observed with the Spy and Nme sgRNA vectors, we observed a substantial level of the unprocessed $\sim 175$-nt tRNA:sgRNA precursor fusion transcript in all cases.

It seemed possible that the relatively inefficient processing observed with these tRNA:sgRNA expression vectors was due either to the shared variable region of the sgRNA present in all these constructs, which is specific for the HSV-1 ICP0 gene, or represented a more general problem of incompatibility of the scaffold region of Sau Cas9-specific sgRNAs with this RNA processing pathway. To determine if either of these hypotheses was correct, we tested two additional tRNA:sgRNA expression constructs in which the ICP0-specific sequence was replaced with variable regions specific for the HSV-1 ICP4 gene or the surface antigen gene of hepatitis B virus (HBV). As may be observed in Figure 3C, both of these Sau-specific sgRNAs were efficiently released from the $\mathrm{tRNA}_{\mathrm{GLN} 1}$ moiety by $\mathrm{tRNase} \mathrm{Z}$ cleavage, and we therefore conclude that it is the ICP0-specific variable region that caused the processing problem seen in Figure 3B. We note that the HSV-1 ICP0-specific variable region used here is very $\mathrm{G}: \mathrm{C}$ rich $(75 \% \mathrm{G}+\mathrm{C})$, and this could certainly inhibit tRNase $Z$ processing by inducing misfolding of the tRNA: sgRNA fusion transcript. The HSV-1 ICP4-specific variable regions used in Figure 2C, lane 6, and Figure 3C, lane 3, are also quite $\mathrm{G}: \mathrm{C}$ rich ( $88 \%$ and $79 \% \mathrm{G}+\mathrm{C}$, respectively) and we again saw a detectable level of the unprocessed tRNA:sgRNA precursor in both cases. In contrast, the other sgRNA variable regions tested generally had lower total levels of $\mathrm{G}+\mathrm{C}$ and were processed efficiently. Therefore, it appears that high $(>70 \%) \mathrm{G}+\mathrm{C}$ levels should be avoided in the sgRNA variable regions of tRNA:sgRNA fusion transcript expression vectors.

The readily detectable level of the ICP0-specific tRNA: sgRNA fusion transcript noted in Figure 3B,C prompted us to consider that this longer transcript might, in fact, be loaded into the Sau Cas9 protein and thereby stabilized. In fact, sgRNAs transcribed by the U6 promoter in the absence of Cas 9 are highly labile and cannot be detected by Northern blot (Fig. 3D, cf. lanes 1 and 2). As expected, the identical ICP0-specific sgRNA, when transcribed by $\mathrm{tRNA}_{\mathrm{GLN} 1}$, was also rapidly degraded in the absence of the cognate Sau Cas 9 protein (Fig. 3D, cf. lanes 3 and 4). Interestingly, instability in the absence of Cas9 was also observed for the longer tRNA:sgRNA precursor transcript, thus demonstrating that Sau Cas9 is able to bind an sgRNA with a long $5^{\prime}$ extension formed by the tRNA moiety. However, we do not presently know whether Cas9 proteins loaded with such an elongated sgRNA are functional.

As the Sau Cas9 protein has not yet been widely used, we also wanted to confirm that cleavage by this endonuclease was occurring at the predicted site located $5^{\prime}$ to the Sau Cas9 PAM sequence $5^{\prime}$-NNGAGT- $3^{\prime}$ used in this plasmid, and that cleavage resulted in the predicted indel mutations after repair by error prone nonhomologous end joining. For this purpose, we used the Surveyor ssDNA-specific nuclease to detect indels in plasmid DNA that was recovered from cells transfected as described in Figure 3A. This DNA was subjected to PCR amplification using primers that generated a 778bp DNA fragment that encompasses the predicted Sau Cas9 cleavage site. As shown in Supplemental Figure S2, we observed readily detectable levels of the predicted DNA fragments of $\sim 548$ and $\sim 230$ bp upon Surveyor treatment of DNA recovered from cultures cotransfected with the Sau Cas/sgRNA expression vector, but not from the control cultures.

One potential concern with the expression of tRNA: sgRNA fusion transcripts, that are then processed by tRNaseZ to liberate the sgRNA, is that this could result in substantially changed tRNA expression levels, either overexpression of the tRNA used in the expression vector or reduced expression if the level of tRNaseZ available in the cell was saturated. To address this potential concern, we generated lentiviral expression vectors encoding Spy Cas9 and a 
puromycin resistance gene as well as an sgRNA transcribed from either the U6 or tRNA $\mathrm{GLN}_{1}$ promoter. The sgRNA used was specific for the HPV18 E6 gene, as described in Figure 1. After packaging, we transduced 293T cells with each of these lentiviral vectors and selected for puromycin resistance. The resultant polyclonal cell lines were expanded and then transiently transfected with the cognate E6 indicator plasmid to confirm that the sgRNA was expressed at a functional level. As shown in Figure 4A, we indeed observed a specific and comparable reduction in the level of FLuc expression, when compared with the RLuc internal control, in both of the transduced cell lines when compared with control 293T cells. Next, we isolated total small RNA from the two transduced cell lines and used deep sequencing to determine the level of expression of all tRNA species in these cells. The resultant data are presented in Figure $4 \mathrm{~B}$ as tRNA copies per cell for each of the 20 tRNA families specific for each amino acid, under the assumption that there are a total of $\sim 2 \times$ $10^{6}$ tRNA molecules per cell (Waldron and Lacroute 1975). As may be observed, we did not detect a significant difference in the expression level of any tRNA, including the glutamine tRNA used to express the sgRNA.

\section{DISCUSSION}

A key current roadblock to the application of CRISPR/Casbased DNA editing technology to the treatment of human disease is the effective and specific delivery of vectors expressing Cas 9 and the appropriate $\operatorname{sgRNA}(\mathrm{s})$ to the appropriate diseased tissue. While delivery using nanoparticles or other
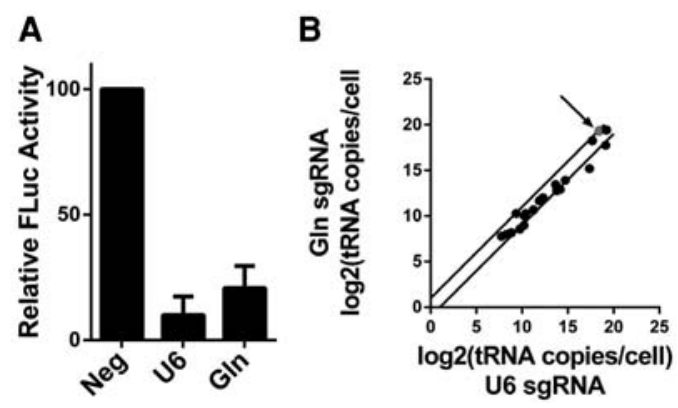

FIGURE 4. Using a tRNA promoter to express an sgRNA does not perturb endogenous tRNA expression levels. (A) Relative FLuc expression in wild-type 293T cells or transduced 293T cells expressing Spy Cas9 and an sgRNA targeting HPV-18 E6 from either the U6 or tRNA $\mathrm{GLN}_{1}$ promoter after transfection with an indicator plasmid containing a cognate HPV-18 E6 target sequence. An RLuc expression vector was also cotransfected as an internal control. Average of three independent experiments with SD indicated. (B) Scatterplot of endogenous tRNA expression levels measured by deep sequencing from cells stably transduced with a lentiviral vector expressing Spy Cas9 as well as an sgRNA from either the U6 or tRNA $\mathrm{GLN}_{1}$ promoter. $\mathrm{tRNAs}$ are grouped by families specific for a particular amino acid. Lines represent a twofold change and the arrow points to the gray dot representing the $\mathrm{RNA}_{\mathrm{GLN}}$ population. For illustrative purposes, this plot assumed there are $2 \times 10^{6}$ tRNA molecules per cell (Waldron and Lacroute 1975). chemical approaches might prove feasible in the future, the only approaches available currently rely on the use of viral vectors. While a number of these exist, AAV stands out as the vector of choice due to its safety and due to the very high titers that can be achieved (Kotterman and Schaffer 2014). However, the small $\sim 4.4$-kb packaging capacity for exogenous DNA represents a major limitation, especially given the large size of both the prototypic Spy Cas9 protein and of the promoters currently used to drive Cas9 and sgRNA expression. Moreover, given the increasing evidence that wild-type Cas 9 can cleave a significant number of off-target DNA sequences in human cells (Frock et al. 2015; Tsai et al. 2015; Wang et al. 2015), it appears likely that the clinical use of Cas9 will require the use of a Cas 9 nickase programmed by two sgRNAs that direct Cas 9 to two closely adjacent targets on opposite strands of the genome, as this approach doubles the target specificity of Cas 9 and can totally suppress off-target cleavages (Mali et al. 2013a; Ran et al. 2013; Frock et al. 2015).

Here, we report that the very short, 70-bp Pol III promoters present in several wild-type human tRNAs are able to express high levels of functional sgRNAs specific for not only a range of DNA targets but also for not only the Spy Cas 9 protein but also the smaller Cas 9 proteins encoded by N. meningitidis and S. aureus, which are only $\sim 3.2 \mathrm{~kb}$ in size. Therefore, using tRNA promoters, it is possible to express two full-length sgRNAs using only $\sim 350$ bp of coding space, leaving $>800$ bp of available space for the Pol II-dependent promoter, NLS, and poly(A) addition site required for Cas9 transcription and function. We therefore believe that, while the larger U6 promoter is fully adequate for the expression of sgRNAs when using plasmids or larger viral vectors, tRNA-derived promoters have the potential to prove invaluable in ongoing efforts to deliver fully active Cas9/sgRNA combinations to cells in vivo using AAV-based vectors. We note that Xie et al. (2015) very recently described the use of the two tRNA processing enzymes RNase $\mathrm{P}$ and tRNase $\mathrm{Z}$ to facilitate the expression of multiplexed sgRNAs in plant cells. However, this proposed technique does not use tRNA promoters to drive sgRNA transcription and requires two complete tRNAs to define both the $5^{\prime}$ and the $3^{\prime}$ end of each sgRNA, rather than only one tRNA as reported here.

\section{MATERIALS AND METHODS}

\section{CRISPR/Cas9 constructs, tRNA, and sgRNA design}

Human tRNA sequences were obtained from the Genomic tRNA database (http://gtrnadb.ucsc.edu) and the MHV68 tRNA M1-7 sequence was obtained from Bogerd et al. (2010). To generate tRNAsgRNA expression constructs, overlapping oligonucleotides were assembled to create a tRNA fused to an sgRNA scaffold and a Pol III termination signal ( $5^{\prime}$-AAAAA- $\left.3^{\prime}\right)$. Between the tRNA and the sgRNA scaffold, BsmBI, BbsI, or BsaI restriction enzyme sites were incorporated to allow for the facile insertion of targeting 
sequences. tRNA-sgRNA cassettes were then cloned into the Cas9 expression vectors pCMVSau, pCMVNme, or pX330 (Addgene, plasmid \#42230) (Cong et al. 2013). pCMVSau expresses human codon optimized Sau Cas9, linked to a FLAG epitope tag and an NLS, under the control of human cytomegalovirus immediate early (CMV-IE) promoter/enhancer. pCMVNme is identical to pCMVSau except that the Sau Cas9 open reading frame was exchanged for a human codon optimized Nme Cas9 gene (Esvelt et al. 2013; Hou et al. 2013). $5^{\prime}$ to this expression cassette, an sgRNA linked to either a U6 promoter or tRNA was inserted. In the case of Spy Cas9 expression vector pX330 (Cong et al. 2013), the U6 promoter was exchanged for the tRNA-sgRNA cassette.

\section{Cell culture}

293T cells were grown in Dulbecco's modified Eagle medium (DMEM) supplemented with $10 \%$ fetal bovine serum, $2 \mathrm{mM}$ antibiotic-antimycotic (Gibco Cell Culture), and $50 \mu \mathrm{g} / \mathrm{mL}$ gentamicin (Life Technologies) at $37^{\circ} \mathrm{C}$.

\section{Reporter assays}

293T cells were plated at $1.25 \times 10^{5}$ cells per well in 12-well plates and transfected using the calcium phosphate method (Cullen 1987) with $1 \mu \mathrm{g}$ of the Cas9/sgRNA expression plasmid, $250 \mathrm{ng}$ of an FLuc-based indicator plasmid, and 10 ng of an RLuc-based control plasmid. Transfected cells were harvested 72-h post-transfection in Passive Lysis Buffer (Promega) and assayed for FLuc and RLuc activity (Promega Dual-Luciferase Reporter Assay System). For the Spy and Nme reporter assays, both a nonspecific sgRNA and an sgRNA specific for the indicator plasmid was included for each tRNA and U6 promoter. Reporter assays for 293T cells stably transduced with lentiviral vectors expressing Cas9 and an sgRNA from either a U6 or tRNA promoter were performed similarly except that cells were only transfected with $50 \mathrm{ng}$ of the FLuc-based indicator plasmid, and $10 \mathrm{ng}$ of an RLuc-based internal control plasmid.

\section{Northern blot assays}

293 T cells were plated at $5.25 \times 10^{6}$ cells in 10 -cm dishes and transfected with $20 \mu \mathrm{g}$ of a Cas $9 / \mathrm{sgRNA}$ expression plasmid using polyethylenimine. Cells were harvested $72-\mathrm{h}$ post-transfection in TRIzol (Life Technologies). Total RNA was isolated and fractionated on a $10 \%$ TBE-Urea Gel (Bio-Rad) and then transferred to HyBond$\mathrm{N}$ membrane (Amersham) and UV crosslinked (Stratalinker, Stratagene). Membranes were prehybridized in ExpressHyb (Clontech) and then incubated at $37^{\circ} \mathrm{C}$ with a ${ }^{32} \mathrm{P}$-end labeled oligonucleotide. Membranes were washed with 2 X SSC $/ 0.1 \%$ SDS at $37^{\circ} \mathrm{C}$ and subjected to autoradiography.

\section{Surveyor assays}

$293 \mathrm{~T}$ cells were plated at $1.25 \times 10^{5}$ cells per well in 12 -well plates and transfected using calcium phosphate, as described above. Total DNA was extracted at $72 \mathrm{~h}$ post-transfection using a DNeasy kit (Qiagen), according to the manufacturer's protocol, and digested with DpnI at $37^{\circ} \mathrm{C}$ for $1 \mathrm{~h}$ to remove input plasmid DNA. The region surrounding the target site on the indicator plasmid was then PCR amplified using the GoTaq cocktail (catalog no. 9PIM300, Promega). Primers used for amplification were as follows: forward primer (5'-CCAGCTACAGTCGGAAACCATC-3'), reverse primer (5'-GAGGTAGATGAGATGTGACGAAC-3'). PCR products were then denatured and reannealed to enable DNA heteroduplex formation as follows: $95^{\circ} \mathrm{C}$ for $10 \mathrm{~min}, 95^{\circ} \mathrm{C}-85^{\circ} \mathrm{C}$ with ramping at $-1^{\circ} \mathrm{C} / \mathrm{sec}, 85^{\circ} \mathrm{C}-25^{\circ} \mathrm{C}$ with ramping at $-0.25^{\circ} \mathrm{C} / \mathrm{sec}$, and a hold at $25^{\circ} \mathrm{C}$ for $1 \mathrm{~min}$. After reannealing, products were treated with Surveyor nuclease and Surveyor enhancer S (Integrated DNA Technologies), according to the manufacturer's protocol, and analyzed on a $2 \%$ agarose gel.

\section{Cell transductions}

The Spy Cas9/sgRNA-expressing lentiviral vectors used were based on lentiCRISPR v2 (Sanjana et al. 2014) (Addgene, plasmid \#52961), except that the U6 promoter was replaced with a glutamine tRNA. An sgRNA targeting the HPV-18 E6 gene was inserted $3^{\prime}$ to the U6 promoter or the glutamine tRNA. 293T cells $\left(2 \times 10^{5}\right)$ were transduced with these lentiviral vectors and then selected with puromycin at $1 \mu \mathrm{g} / \mathrm{mL}$ for $72 \mathrm{~h}$ after transduction. The resultant polyclonal cell lines were then maintained in media containing puromycin.

\section{Deep-sequencing analysis}

Total RNA present in the transduced 293T cells was harvested and small RNAs $(<250 \mathrm{nt})$ isolated using a mirVana miRNA isolation kit (Ambion). Small RNAs between $\sim 50$ and $120 \mathrm{nt}$ were further isolated by size fractionating after polyacrylamide gel electrophoresis using 10\% Tris-borate-EDTA (TBE)-urea gels (Bio-Rad) followed by electroelution. To ensure the presence of a single $5^{\prime}$ phosphate, the isolated RNAs were dephosphorylated using recombinant shrimp alkaline phosphatase (NEB) in NEB cutsmart buffer. The reaction was heated at $65^{\circ} \mathrm{C}$ for $5 \mathrm{~min}$ and then the RNA molecules were phosphorylated using T4 polynucleotide kinase (NEB) in NEB Ligase Buffer. The reaction was then incubated at $65^{\circ} \mathrm{C}$ for 20 min. The isolated small RNAs were then processed using a TruSeq small RNA sample preparation kit (Illumina). cDNA was generated as previously described (Flores et al. 2014). Briefly, adapter-ligated RNA was reverse transcribed using SuperScript III (Life Technologies) and amplified using GoTaq green PCR master mix (Promega) with TruSeq $3^{\prime}$ indices. cDNA molecules corresponding to a length of $\sim 170-240$ bp were then isolated by gel electrophoresis and sequenced on an Illumina HiSeq 2000 using a 50-bp single end protocol.

Bioinformatic analyses were completed as follows. Reads were first processed with the FASTX-Toolkit and Bowtie as described previously (Flores et al. 2014). Briefly, reads $>15$ nt were collapsed into FASTA format with the FASTX-Toolkit (http://hannonlab.cshl.edu/ fastx_toolkit/index.html) using the following pipeline: fastq_quality_filter -Q 33 | fastq_to_fasta -Q 33 | fastx_clipper -a TruSeqIndex\# -1 15 | fastx_collapser. Note that reads with and without the $3^{\prime}$ adapter were retained. All reads were then subject to alignment using Bowtie v.0.12.7 (Langmead et al. 2009) with the following options: $-\mathrm{k} 1$ (default) $-\mathrm{v} 1$-best - strata $-\mathrm{m} 25$. The reads were sequentially aligned and filtered to adapter sequences, miRBase v. 20 annotations of miRNA hairpins, (Kozomara and Griffiths-Jones 2014), and finally the human functional RNA database version 3.4 
(Mituyama et al. 2009). Reads aligning to the fRNAdb were further filtered with in-house Perl scripts. All reads $>25 \mathrm{nt}$ aligning to all tRNAs in the fRNAdb were assigned unambiguously to an amino acid group and then summed. These read totals were then normalized both to total reads aligning to the fRNAdb to assess tRNA pool sizes and internally to assess relative pool stoichiometry. As a scaling factor, it was assumed there are $2 \times 10^{6}$ tRNA molecules per cell (Waldron and Lacroute 1975).

\section{DATA DEPOSITION}

The raw sequencing data obtained from small RNA deep sequencing have been submitted to the NCBI Gene Expression Omnibus (GEO) (http://www.ncbi.nlm.nih.gov/geo/) under accession number GSE69720.

\section{SUPPLEMENTAL MATERIAL}

Supplemental material is available for this article.

\section{ACKNOWLEDGMENTS}

This research was supported in part by the Duke University Center for AIDS Research (CFAR), a National Institutes of Health-funded program (5P30 AI064518), and by grants from the Farrah Fawcett Foundation and F. Hoffman-LaRoche. A.L.M. was supported by T32-CA009111 from the National Institutes of Health.

Received March 4, 2015; accepted June 12, 2015.

\section{REFERENCES}

Bogerd HP, Karnowski HW, Cai X, Shin J, Pohlers M, Cullen BR. 2010. A mammalian herpesvirus uses noncanonical expression and processing mechanisms to generate viral microRNAs. Mol Cell 37: 135-142.

Cong L, Ran FA, Cox D, Lin S, Barretto R, Habib N, Hsu PD, Wu X, Jiang W, Marraffini LA, et al. 2013. Multiplex genome engineering using CRISPR/Cas systems. Science 339: 819-823.

Cox DB, Platt RJ, Zhang F. 2015. Therapeutic genome editing: prospects and challenges. Nat Med 21: 121-131.

Cullen BR. 1987. Use of eukaryotic expression technology in the functional analysis of cloned genes. Methods Enzymol 152: 684-704.

Esvelt KM, Mali P, Braff JL, Moosburner M, Yaung SJ, Church GM. 2013. Orthogonal Cas9 proteins for RNA-guided gene regulation and editing. Nat Methods 10: 1116-1121.

Flores O, Kennedy EM, Skalsky RL, Cullen BR. 2014. Differential RISC association of endogenous human microRNAs predicts their inhibitory potential. Nucleic Acids Res 42: 4629-4639.

Frock RL, Hu J, Meyers RM, Ho YJ, Kii E, Alt FW. 2015. Genome-wide detection of DNA double-stranded breaks induced by engineered nucleases. Nat Biotechnol 33: 179-186.

Hou Z, Zhang Y, Propson NE, Howden SE, Chu LF, Sontheimer EJ, Thomson JA. 2013. Efficient genome engineering in human pluripotent stem cells using Cas9 from Neisseria meningitidis. Proc Natl Acad Sci 110: 15644-15649.
Hsu PD, Lander ES, Zhang F. 2014. Development and applications of CRISPR-Cas9 for genome engineering. Cell 157: 1262-1278.

Kennedy EM, Kornepati AV, Goldstein M, Bogerd HP, Poling BC, Whisnant AW, Kastan MB, Cullen BR. 2014. Inactivation of the human papillomavirus E6 or E7 gene in cervical carcinoma cells by using a bacterial CRISPR/Cas RNA-guided endonuclease. J Virol 88: 11965-11972.

Kennedy EM, Bassit LC, Mueller H, Kornepati AVR, Bogerd HP, Nie T, Chatterjee P, Javanbakht H, Schinazi RF, Cullen BR. 2015. Suppression of hepatitis B virus DNA accumulation in chronically infected cells using a bacterial CRISPR/Cas RNA-guided DNA endonuclease. Virology 476: 196-205.

Kotterman MA, Schaffer DV. 2014. Engineering adeno-associated viruses for clinical gene therapy. Nat Rev Genet 15: 445-451.

Kozomara A, Griffiths-Jones S. 2014. miRBase: annotating high confidence microRNAs using deep sequencing data. Nucleic Acids Res 42: D68-D73.

Langmead B, Trapnell C, Pop M, Salzberg SL. 2009. Ultrafast and memory-efficient alignment of short DNA sequences to the human genome. Genome Biol 10: R25.

Mali P, Aach J, Stranges PB, Esvelt KM, Moosburner M, Kosuri S, Yang L, Church GM. 2013a. CAS9 transcriptional activators for target specificity screening and paired nickases for cooperative genome engineering. Nat Biotechnol 31: 833-838.

Mali P, Yang L, Esvelt KM, Aach J, Guell M, DiCarlo JE, Norville JE, Church GM. 2013b. RNA-guided human genome engineering via Cas9. Science 339: 823-826.

Mituyama T, Yamada K, Hattori E, Okida H, Ono Y, Terai G, Yoshizawa A, Komori T, Asai K. 2009. The functional RNA database 3.0: databases to support mining and annotation of functional RNAs. Nucleic Acids Res 37: D89-D92.

Ran FA, Hsu PD, Lin CY, Gootenberg JS, Konermann S, Trevino AE, Scott DA, Inoue A, Matoba S, Zhang Y, et al. 2013. Double nicking by RNA-guided CRISPR Cas9 for enhanced genome editing specificity. Cell 154: 1380-1389.

Ran FA, Cong L, Yan WX, Scott DA, Gootenberg JS, Kriz AJ, Zetsche B, Shalem O, Wu X, Makarova KS, et al. 2015. In vivo genome editing using Staphylococcus aureus Cas9. Nature 520: 186-191.

Sanjana NE, Shalem O, Zhang F. 2014. Improved vectors and genomewide libraries for CRISPR screening. Nat Methods 11: 783-784.

Swiech L, Heidenreich M, Banerjee A, Habib N, Li Y, Trombetta J, Sur M, Zhang F. 2015. In vivo interrogation of gene function in the mammalian brain using CRISPR-Cas9. Nat Biotechnol 33: $102-106$.

Tsai SQ, Zheng Z, Nguyen NT, Liebers M, Topkar VV, Thapar V, Wyvekens N, Khayter C, Iafrate AJ, Le LP, et al. 2015. GUIDE-seq enables genome-wide profiling of off-target cleavage by CRISPRCas nucleases. Nat Biotechnol 33: 187-197.

Waldron C, Lacroute F. 1975. Effect of growth rate on the amounts of ribosomal and transfer ribonucleic acids in yeast. $J$ Bacteriol 122: 855-865.

Wang X, Wang Y, Wu X, Wang J, Wang Y, Qiu Z, Chang T, Huang H, Lin RJ, Yee JK. 2015. Unbiased detection of off-target cleavage by CRISPR-Cas9 and TALENs using integrase-defective lentiviral vectors. Nat Biotechnol 33: 175-178.

Xie K, Minkenberg B, Yang Y. 2015. Boosting CRISPR/Cas9 multiplex editing capability with the endogenous tRNA-processing system. Proc Natl Acad Sci 112: 3570-3575.

Zhang Y, Heidrich N, Ampattu BJ, Gunderson CW, Seifert HS, Schoen C, Vogel J, Sontheimer EJ. 2013. Processing-independent CRISPR RNAs limit natural transformation in Neisseria meningitidis. Mol Cell 50: 488-503. 

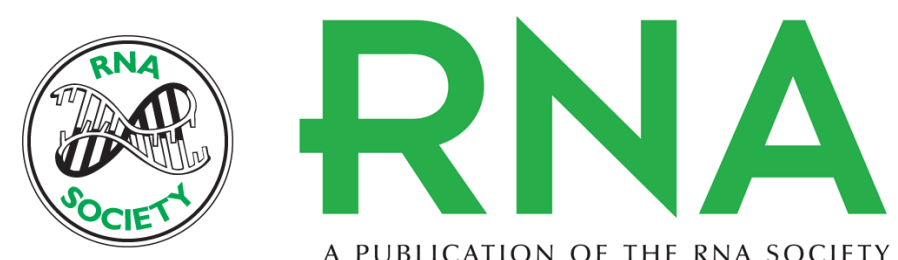

A PUBLICATION OF THE RNA SOCIETY

\title{
Expression of CRISPR/Cas single guide RNAs using small tRNA promoters
}

\author{
Adam L. Mefferd, Anand V.R. Kornepati, Hal P. Bogerd, et al. \\ RNA 2015 21: 1683-1689 originally published online July 17, 2015 \\ Access the most recent version at doi:10.1261/rna.051631.115
}

\section{Supplemental http://rnajournal.cshlp.org/content/suppl/2015/07/10/rna.051631.115.DC1 Material}

References This article cites 26 articles, 6 of which can be accessed free at: http://rnajournal.cshlp.org/content/21/9/1683.full.html\#ref-list-1

Creative This article is distributed exclusively by the RNA Society for the first 12 months after the Commons License full-issue publication date (see http://rnajournal.cshlp.org/site/misc/terms.xhtml). After 12 months, it is available under a Creative Commons License (Attribution-NonCommercial 4.0 International), as described at http://creativecommons.org/licenses/by-nc/4.0/.
Email Alerting Receive free email alerts when new articles cite this article - sign up in the box at the Service top right corner of the article or click here.

